\title{
Growing oocyte-specific transcription-dependent de novo DNA methylation at the imprinted Zrsr1-DMR
}

\author{
Keiichiro Joh ${ }^{*}$ (0), Fumikazu Matsuhisa ${ }^{2 \dagger}$, Shuji Kitajima ${ }^{2 \dagger}$, Kenichi Nishioka ${ }^{1}$, Ken Higashimoto ${ }^{1}$, Hitomi Yatsuki ${ }^{1}$,
} Tomohiro Kono ${ }^{3}$, Haruhiko Koseki ${ }^{4}$ and Hidenobu Soejima ${ }^{{ }^{*}}$

\begin{abstract}
Background: Zrsr1 is a paternally expressed imprinted gene located in the first intron of Commd1, and the Zrsr1 promoter resides in a differentially methylated region (DMR) that is maternally methylated in the oocyte. However, a mechanism for the establishment of the methylation has remained obscure. Commd 1 is transcribed in the opposite direction to Zrsr1 with predominant maternal expression, especially in the adult brain.

Results: We found Commed 1 transcribed through the DMR in the growing oocyte. Zrsr1-DMR methylation was abolished by the prevention of Commd1 transcription. Furthermore, methylation did not occur at the artificially unmethylated maternal Zrsr1-DMR during embryonic development when transcription through the DMR was restored in the zygote. Loss of methylation at the maternal Zrsr1-DMR resulted in biallelic Zrsr1 expression and reduced the extent of the predominant maternal expression of Commd1.

Conclusions: These results indicate that the establishment of methylation at Zrsr1-DMR occurs in a transcriptiondependent and oocyte-specific manner and caused Zrsr1 imprinting by repressing maternal expression. The predominant maternal expression of Commd1 is likely caused by transcriptional interference by paternal Zrsr 1 expression.
\end{abstract}

Keywords: DMR, DNA methylation, Genomic imprinting

\section{Background}

Genomic imprinting is an epigenetic phenomenon of parent-of-origin-dependent expression that is observed in a subset of mammalian genes. Imprinted genes are expressed exclusively or predominantly from one of the two parental alleles and are frequently located in clusters known as imprinted domains. The expression of genes in an imprinted domain is regulated by a discrete element called an imprinting center (IC) or an imprinting control region (ICR). Imprinted genes or imprinted domains are associated with differentially methylated regions (DMRs) that exhibit parent-of-origin-specific DNA methylation.

\footnotetext{
*Correspondence: joh@cc.saga-u.ac.jp; soejimah@cc.saga-u.ac.jp

${ }^{\dagger}$ Fumikazu Matsuhisa and Shuji Kitajima contributed equally to this work

${ }^{1}$ Division of Molecular Genetics and Epigenetics, Department of Biomolecular Sciences, Faculty of Medicine, Saga University,

Saga 849-8501, Japan

Full list of author information is available at the end of the article
}

Two classes of DMRs have been identified as follows: germline DMRs (gDMRs), or primary DMRs; and somatic DMRs (sDMRs), or secondary DMRs. gDMR methylation is established during gametogenesis, and sDMRs acquire methylation after fertilization under the direction of gDMRs. The ICs of the imprinted genes are located in their corresponding gDMRs. More than 20 gDMRs have been identified in mice, of which only three are paternally methylated [1-4]. Recent studies have identified an additional 11 new putative maternally methylated gDMRs [5].

DNA methylation at gDMRs is the primary determinant of the allelic expression of imprinted genes, and the mechanisms of methylation establishment have been extensively investigated. The specific recruitment of de novo methylation machineries to gDMR methylation sites via the recognition of sequence elements and/ or chromatin structures has been considered as a potential mechanism of germline-specific gDMR methylation 
establishment $[1,6]$. However, efforts to identify sequence motifs for gDMR methylation have not been successful. Several trans-acting factors for maternally methylated gDMRs have been found to be essential for the establishment of germline methylation in mice. For example, Dnmt3a has been identified as the enzyme responsible for de novo methylation of many maternal gDMRs [7, 8]. Dnmt3l, a DNA methyltransferase (DNMT)-like protein without enzymatic activity, is the likely co-factor of DNMTs [9]. Ablation of Kdm1b, a histone demethylase of H3K4 di-and trimethylation, in oocytes resulted in the failure of methylation establishment at some maternal gDMRs [10]. In addition, the deletion of Hira, which encodes a histone H3.3 chaperon (Hira), led to global hypomethylation in oocytes [11].

Kelsey et al. proposed a model for the establishment of methylation at the maternal gDMRs in the oocyte [12, 13], which suggests that maternal methylation of gDMRs is regulated by the same mechanisms of general genebody methylation reported for active genes $[14,15]$. This was based on the findings that most maternal gDMRs are located in actively transcribed regions [16], that transcription is a prerequisite for the establishment of methylation at four maternal gDMRs [13, 16-18], and regarding the characteristics of the methylome and transcriptome in growing oocytes $[13,19]$. Analyses of the methylome revealed that most methylation in the oocyte genome occurs within actively transcribed regions and that maternal gDMRs are not specifically targeted for methylation, but are instead methylated along with other parts of the transcribed regions where the gDMRs reside. Unlike the rest of the transcribed regions, gDMRs are likely protected against global demethylation during early embryonic development; only the gDMRs escape global demethylation and remain methylated throughout development.

Methylation failed to be established at the gDMRs in the Gnas locus and the KvDMR in the Kcnq1 locus when a poly(A) signal truncation cassette was inserted into these loci to prevent transcription from elongation through the gDMRs $[16,18]$. Failure of methylation was also reported at PWS-IC and the Zac1-DMR when the promoter regions from which transcription originated and then proceeded through the maternal gDMRs were deleted [13, 17]. Furthermore, most of these maternal gDMRs were located within transcribed regions in the growing oocyte [16]. On the other hand, the establishment of sDMR- and lineage-specific methylation during the post-implantation stage clearly indicates the de novo methylation potency of early embryonic cells, including naïve/primed pluripotent stem cells [20]. However, it is unknown whether de novo methylation occurs at gDMRs during the post-implantation stage after failure to establish gDMR methylation in the oocyte.
Zrsr1 (U2af1-rs1) and Commd1 (Murr1) are imprinted protein coding genes located in mouse proximal chromosome 11. Zrsr1 is expressed ubiquitously in all adult tissues examined and is expressed exclusively from the paternal allele. Zrsr1 resides in the first intron of the Commd1 gene and is transcribed in the opposite direction to the host gene. The Zrsr1 promoter is located in a maternally methylated gDMR, i.e., the Zrsr1-DMR [21]. It is likely that maternal methylation at the Zrsr1-DMR causes imprinted expression by repressing maternal expression of the gene. Commd1 is likewise expressed ubiquitously in adult mice, but is expressed from both parental alleles. However, Commd1 expression from the maternal allele is stronger than expression from the paternal allele (i.e., predominant maternal expression), as exemplified in the adult brain. Although Zrsr1-DMR resides in the transcribed region of Commd1, the link between transcription and the establishment of methylation at this DMR has not been clarified.

In this study, we found that methylation at Zrsr1-DMR failed to be established when transcription through the DMR was abolished by the insertion of a poly(A) signal cassette into the site between the Commd1 promoter and the Zrsr1 gene. Furthermore, upon deletion of the cassette in the zygote, Zrsr1-DMR transcription resumed, but methylation at the DMR during early development was not restored. These results indicate that transcription-dependent methylation at the DMR occurs specifically in the growing oocyte, but not during early development. We also found that interference with transcription likely caused predominant maternal expression of Commd1 in the adult brain.

\section{Results}

Truncation of Commd 1 transcription results in methylation failure at Zrsr1-DMR in the growing oocyte

Methylation of maternal gDMRs of imprinted genes is established asynchronously during postnatal oocyte growth, typically between 5 and 25 days postpartum (dpp) [22]. To test whether de novo methylation at Zrsr1DMR is dependent on transcription resulting from Commd 1 expression in the oocyte, we analyzed Commd1 expression and the methylation status of Zrsr1-DMR in growing oocytes from this period and in ovulated MII oocytes. Commd1 was expressed in all periods of oocyte maturation analyzed (Fig. 1b). De novo methylation at Zrsr1-DMR started after $10 \mathrm{dpp}$ and was completed between $15 \mathrm{dpp}$ and maturation (Fig. 1c). Thus, transcription through the Zrsr1-DMR proceeded before and during the establishment of methylation. Zrsr1 expression was not detected by RT-PCR during oocyte maturation (Additional file 1: Figure S1).

To determine whether de novo methylation at Zrsr1DMR was dependent on Commd1 elongation through 
a
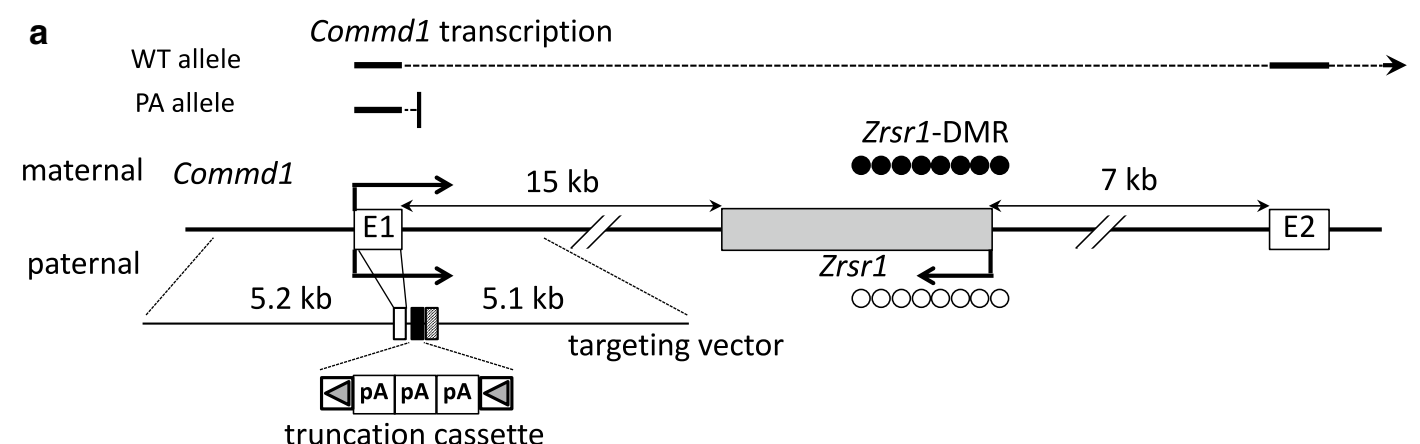

b

D5 D10 D15 MII PC

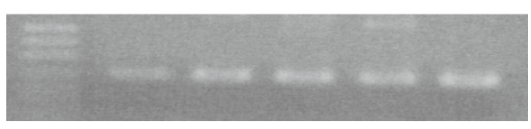

C

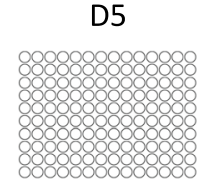

D10

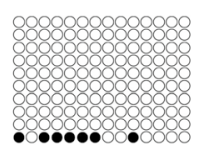

D15

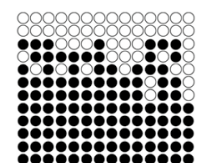

MII

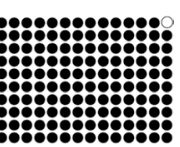

Fig. 1 Structure of the Zrsr1/Commd1 locus and analysis of Commd1 expression and Zrsr1-DMR methylation in the oocyte. a Zrsr1, an approximately 2.8-kb intronless gene, and the first two exons of Commd1 are represented by gray and white boxes, respectively. Distances from the Zrsr 1 gene to Commd 1 exon 1 and exon 2 are indicated above the gene with double-headed arrows. The schematic is not drawn to scale. Arrows above (maternal allele) and below (paternal allele) exon 1 and the Zrsr gene represent the direction of transcription and the allelic expression status of the genes. The open and closed circles at the Zrsr 1 promoter indicate unmethylation and methylation, respectively. A schematic of the targeting vector is shown under the gene. The closed and hatched boxes represent the truncation cassette and the neo-selection marker gene, respectively. These elements are flanked by the $5.2 \mathrm{~kb}$ left arm containing exon 1 and the $5.1 \mathrm{~kb}$ right arm, which contains part of intron 1. The truncation cassette is flanked by loxP sites, represented by gray arrowheads enclosed in open rectangles. Expected transcription patterns of the WT and PA alleles are shown above the gene schematic with thick lines and dotted lines corresponding to exons and introns, respectively. $\mathbf{b}$ RT-PCR analysis of Commd 1 expression in growing oocytes prepared from B6 female neonates at Day 5 (D5), Day 10 (D10), and Day 15 (D15) postpartum, and fully grown MII oocytes (MII) from B6 adult females. PC: positive control for RT-PCR using adult brain CDNA. MW: molecular weight marker. c Analysis of methylation at Zrsr1-DMR in growing and fully grown oocytes used in $\mathbf{b}$. The 223-bp region in the DMR containing $14 \mathrm{CpGs}$ was analyzed via bisulfite sequencing. Each row represents a dataset from one clone, and each circle represents one $\mathrm{CpG}$ site. Closed and open circles depict methylated and unmethylated CpGs, respectively

this DNA element, we inserted a truncation cassette containing three tandem copies of SV40 poly(A) signal into intron 1 of Commd1 to generate the transcription-truncation allele Commd1 ${ }^{P A}$ (Fig. 1a) and obtained Commd $1^{+/ P A}$-heterozygous mice in a C57BL/6 J background. No Commd $1^{P A / P A}$ mice were born from the intercrossing of heterozygous parents. Commd1 $1^{-1-}$ mice have been shown to be embryonically lethal at E9.5 to E10.5 [23]; the absence of homozygous pups for the truncation allele was thus likely attributable to embryonic lethality, which strongly suggests that a truncation occurred as expected and rendered the Commd $1^{P A}$ allele functionally null.

To assess the truncation of the Commd $1^{P A}$ allele and Zrsr1-DMR methylation in the MII oocyte, MII oocytes were obtained from adult F1 females generated from the cross between Commd1 $1^{+/ P A} \mathrm{~B} 6$ females and WT PWK males. Commd1 $1^{P A(B G) /+(P W K)}$ mice, termed PA F1 mice, and Commd1 $1^{+(B) /+(P W K)}$ mice, termed WT F1 mice, were obtained from F1 littermates. The allelic expression of Commd1 was analyzed by RFLP analysis of RT-PCR products with the primers Comm-F1 and Comm-R1, located at exon 1 and exon 2, respectively. Expression of the Commd $1^{P A}$ allele was not detected in MII oocytes prepared from the PA F1 females, although expression of the PWK allele was detected. In contrast, both alleles were expressed in oocytes from WT F1 female littermates (Fig. 2a). The Zrsr1-DMR was completely unmethylated on the truncated allele (B6) in the MII oocytes from the PA F1 females, in contrast to the WT PWK allele, which was completely methylated. As expected, in oocytes from the WT F1 females, Zrsr1DMR was completely methylated on both the B6 and PWK alleles (Fig. 2b). These results indicate that transcription termination occurred in intron 1, likely at the truncation cassette, and resulted in the loss of transcription through the DMR, which led to methylation failure at the DMR during oogenesis.

Maternal methylation at Zrsr1-DMR causes Zrsr1 imprinting To investigate the causative link between the maternal methylation at Zrsr1-DMR and the imprinted 


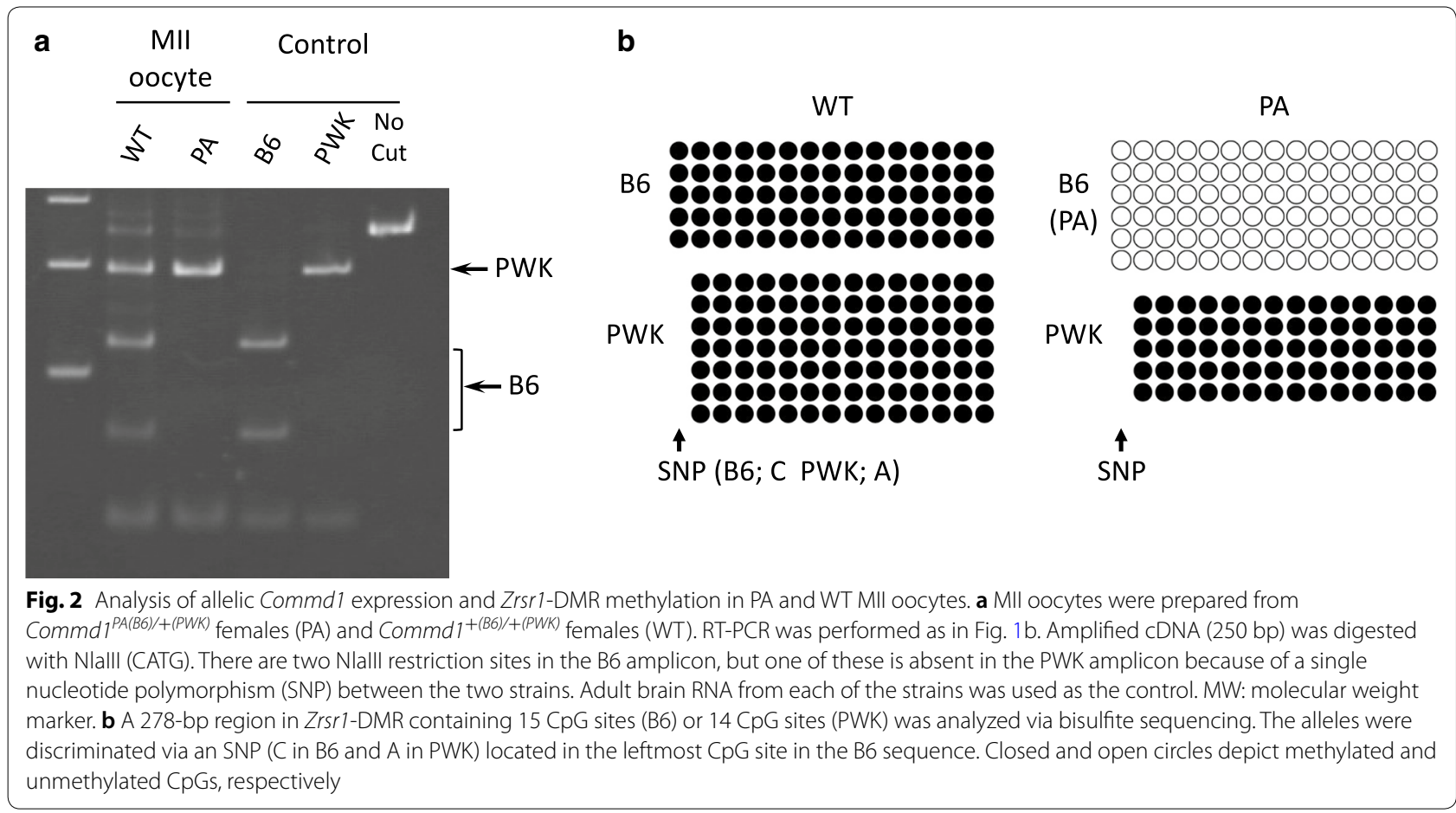

expression of Zrsr1, we analyzed Zrsr1-DMR methylation and Zrsr1 allelic expression in the adult F1 mice described above. The truncation of Commd1 transcription was also observed in the brain and liver of the PA F1 mice (Fig. 3a). The Zrsr1-DMR on the maternal Commd $1^{P A(B 6)}$ allele of the PA F1 mice was completely unmethylated, whereas normal maternal methylation was observed in the WT F1 mice (Fig. 3b). These results indicate that the truncation cassette was also functional in the adult mice and that the unmethylated status of maternal Zrsr1-DMR persisted throughout the embryo stage and postnatal growth to adulthood. Zrsr1 was biallelically expressed in the PA F1 mice, while in WT F1 mice the gene exhibited paternal-specific expression in the WT F1 mice (Fig. 3c). Quantitative analysis of total Zrsr1 mRNA levels indicated that the PA F1 mice expressed twice as much Zrsr1 as the WT F1 mice (Fig. 3d), which suggests that the maternal allele in the PA F1 mice was completely de-repressed and was expressed at the same level as the paternal allele. Thus, it is evident that the Zrsrl gene is imprinted by suppression of maternal allele expression and that maternal methylation at the DMR is the primary imprint mark.

\section{Zygotic deletion of the truncation cassette does} not result in the acquisition of methylation at Zrsr1-DMR during embryonic development and postnatal growth We showed that Zrsr1-DMR acquires methylation in a transcription-dependent manner during oogenesis.
However, Commd1 is expressed from both alleles and paternal Zrsr1-DMR is unmethylated in various tissues of adult mice [21, 24]. This fact indicates that transcription-dependent methylation does not occur at paternal Zrsr1-DMR in differentiated somatic cells. However, it was not known that Commd1 is expressed in early embryo, in which genome-wide de novo methylation is about to occur $[19,25,26]$. Commd1 was expressed from both alleles in the WT blastocysts described below (WT in Fig. 4a, b), which indicated that the paternal Zrsr1DMR was also unmethylated, even during transcription in pluripotent cells. To confirm this in the maternal allele, the floxed truncation cassette (refer to Fig. 1a) was deleted from the maternal Commd $1^{P A}$ allele via zygotic expression of Cre recombinase from CAG-Cre transgene. Then, methylation at the maternal Zrsr1-DMR, which was inherited in an unmethylated state, was analyzed. The resulting deleted allele was denoted Commd $1^{\triangle P A}$.

The blastocysts were prepared via in vitro fertilization (IVF) with MII oocytes from the Commd1 $1^{+/ P A}$ B6 females and sperm from CAG-Cre transgenic males in a $\mathrm{BALB} / \mathrm{c}$ background. Deletion of the truncation cassette occurred in all blastocysts that inherited the Commd $1^{P A}$ allele and the CAG-Cre transgene(s) (Additional file 2: Figure S2). Judging from the high incidence of deletion at the blastocyst stage, it is plausible that the deletion actually occurred much earlier. To determine whether deletion of the truncation cassette would restore expression of the maternal Commd1 allele, we analyzed the allelic 


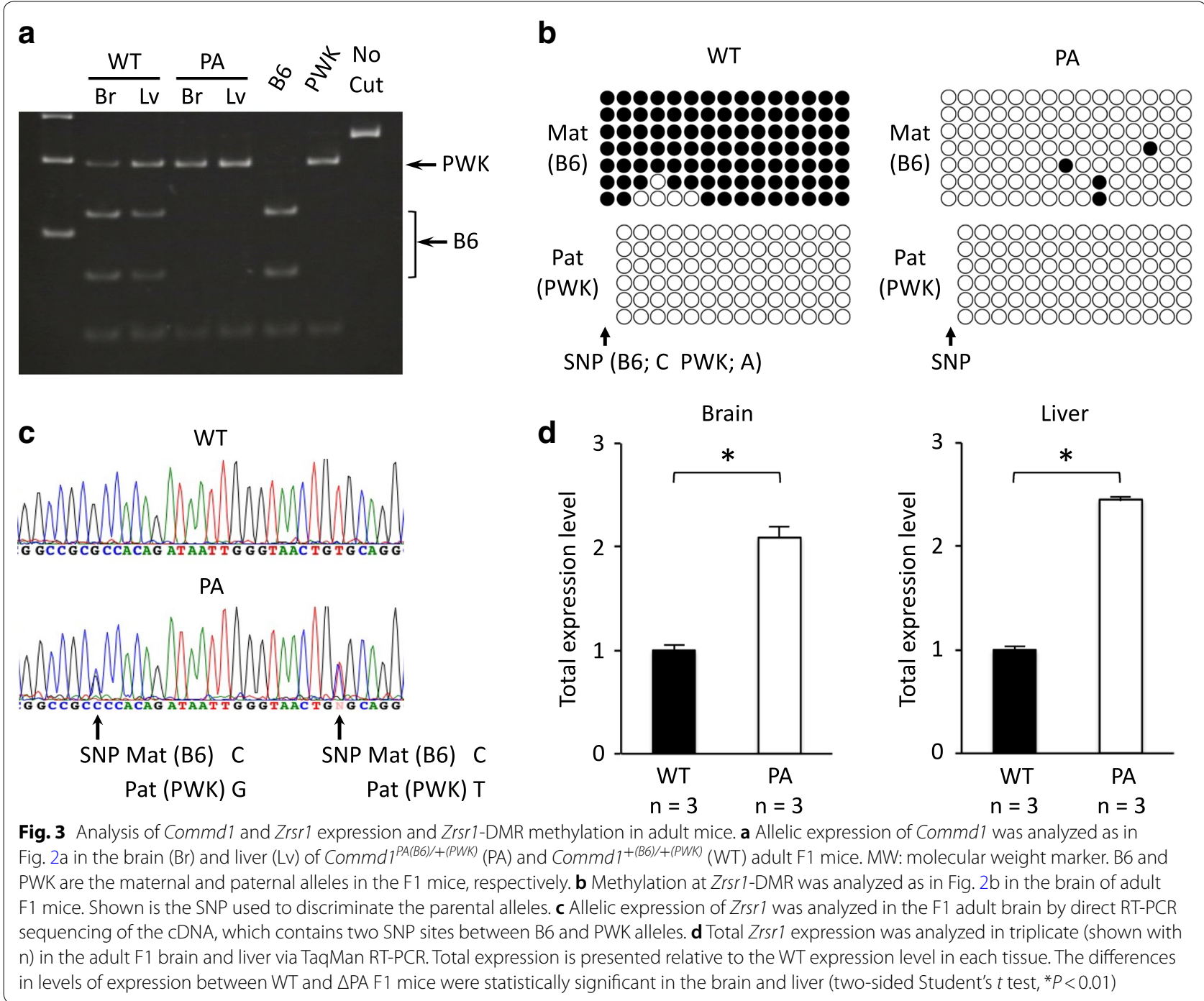

expression of Commd1 in Commd1 $1^{+(B 6) /+(B A L B)}$ blas-

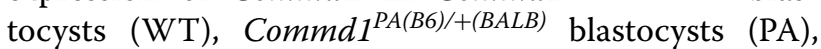
and Commd $1^{\triangle P A(B 6) /+(B A L B)}$ blastocysts ( $\left.\triangle \mathrm{PA}\right)$. Allelic expression was analyzed quantitatively by pyrosequencing of RT-PCR products with the primers CommExpreF1-bio and CommExprePyro-R (Table S1), which are located in exon 2. In the WT blastocysts, Commd1 was equally expressed from both parental alleles, but only the paternal allele was expressed in the PA blastocysts. This indicates that the truncation cassette was also functional in the blastocysts, and suggests that Commd1 was expressed from both alleles during the developmental period in which global de novo DNA methylation occurs. In $\triangle \mathrm{PA}$ blastocysts, in which the truncation cassette was deleted, the maternal $\triangle \mathrm{PA}$ allele was expressed at levels comparable to the paternal allele (Fig. 4a). Furthermore, the unmethylated status of maternal Zrsr1-DMR was maintained, despite transcription of the DMR (Fig. 4b). To determine whether this status was maintained while under transcription from the early embryonic stage to adulthood, we analyzed the brain of $\triangle \mathrm{PA}$ adult $\mathrm{F} 1$ mice from the cross between Commd $1^{+/ P A}$ B6 females and CAG-Cre transgenic BALB/c males. The maternal Zrsr1-DMR in the Commd $1^{\triangle P A}$ allele was unmethylated, even though the maternal allele was expressed (Fig. 4c, d). Thus, we concluded that maternal Zrsr1-DMR was not methylated after fertilization, irrespective of DMR transcription.

\section{Predominant maternal expression of Commd1 is caused by the imprinted expression of Zrsr 1}

We reported predominant maternal expression of Commd1, especially in the brains of adult mice [24] (WT in Fig. 4d, Additional file 3: Figure S3). Based on 


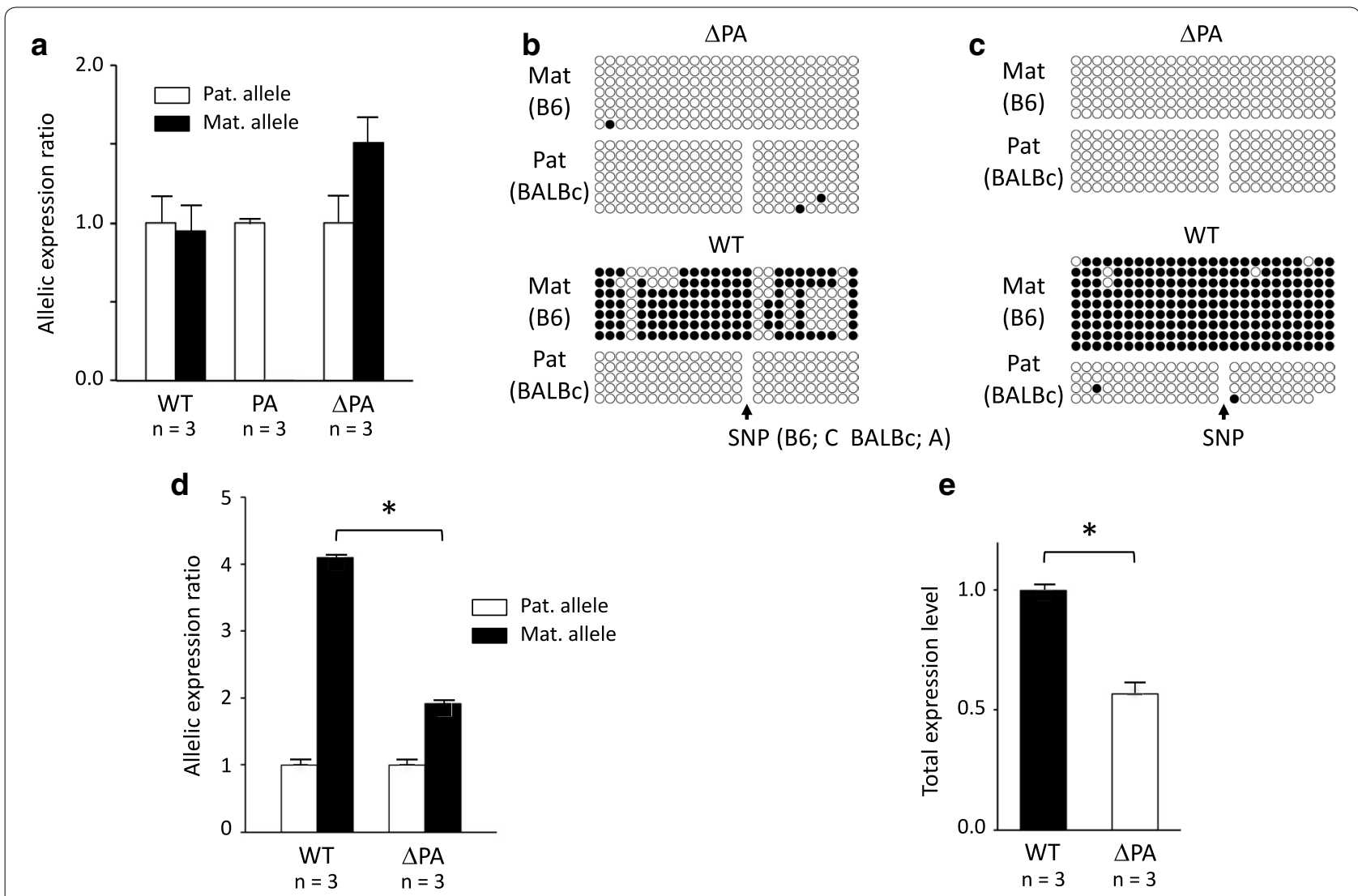

Fig. 4 Quantitative analysis of Commd1 expression and Zrsr1-DMR methylation in $\triangle P A$ blastocysts and the $\triangle P A$ adult brain. a Allelic expression of Commd 1 was analyzed in $\operatorname{Commd1}^{+(B 6) /+(B A L B)}(\mathrm{WT})$, Commd $1^{P A(B G) /+(B A L B)}(\mathrm{PA})$, and $\operatorname{Commd} 1^{\triangle P A(B G) /+(B A L B)}(\triangle \mathrm{PA})$ blastocysts in triplicate (shown with $\mathrm{n}$ ) by pyrosequencing. Alleles were discriminated between using an SNP between B6 (Mat) and BALB/C (Pat) located in exon 2 (rs26846230; C in B6, $\mathrm{T}$ in BALB/C). The allelic expression ratios are presented relative to paternal expression in each blastocyst sample. b Methylation at Zrsr1-DMR was analyzed in the blastocysts used in $\mathbf{a}$. The 343-bp region in the DMR containing $25 \mathrm{CpG}$ sites (B6; maternal allele) or $24 \mathrm{CpG}$ sites (BALB/c; paternal allele) was analyzed via bisulfite sequencing. The alleles were discriminated between using an SNP (rs26846192; C in B6, A in BALB/c) located in a CpG site in the B6 sequence. Closed and open circles depict methylated and unmethylated CpGs, respectively. c Methylation at Zrsr1-DMR was analyzed in the brains of $\triangle P A$ and WT adult F1 mice as described in $\mathbf{b}$. $\mathbf{d}$ Allelic expression of Commd 1 in the adult F1 brain was quantitatively analyzed in triplicate (shown with $\mathrm{n}$ ) by pyrosequencing and is presented as in a. e Total Commd1 expression in an adult F1 brain of each genotype was analyzed in triplicate (shown with n) via TaqMan RT-PCR. Total expression is presented relative to the WT expression level. Asterisks (*) indicate statistical significance $(P<0.01)$ according to the two-sided Student's $t$ test

the two observations [24] discussed below, we hypothesized that the expression of Zrsr1 decreased Commd1 expression from the paternal allele by interfering with the transcription elongation of Commd1 within the gene body and with transcription initiation in the Commd1 promoter. First, in the adult liver, Zrsr 1 is expressed at extremely low levels (less than 10\%) relative to Commd1, which exhibits less pronounced predominant maternal expression (Additional file 3: Figure S3). However, in the adult brain, Zrsr1 is expressed to the same extent as Commd1, which exhibits remarkable predominant maternal expression. Second, the antisense transcript of Commd1 was observed in the promoter region of Commd 1 only in the paternal allele and was observed at higher levels in the brain than in the liver (also refer to [27] and Fig. 5b). The observed antisense Commd 1 transcript is presumably generated by read-through transcription of Zrsr1, which may interfere with the initiation of Commd1 transcription.

According to this hypothesis, we expected that predominant maternal expression would not occur in the $\triangle \mathrm{PA}$ mice, which express Zrsrl equally from both parental alleles. Indeed, quantitative analysis by pyrosequencing demonstrated that the ratio of maternal to paternal expression was significantly decreased in these mice relative to WT mice (Fig. 4d). The total Commd1 expression levels were then quantitatively analyzed in both lines using the TaqMan assay (Fig. 4e). Expression of Commd 1 in the $\triangle \mathrm{PA}$ mice was $57 \%$ of that in the WT mice, which is consistent with the notion of decreased 

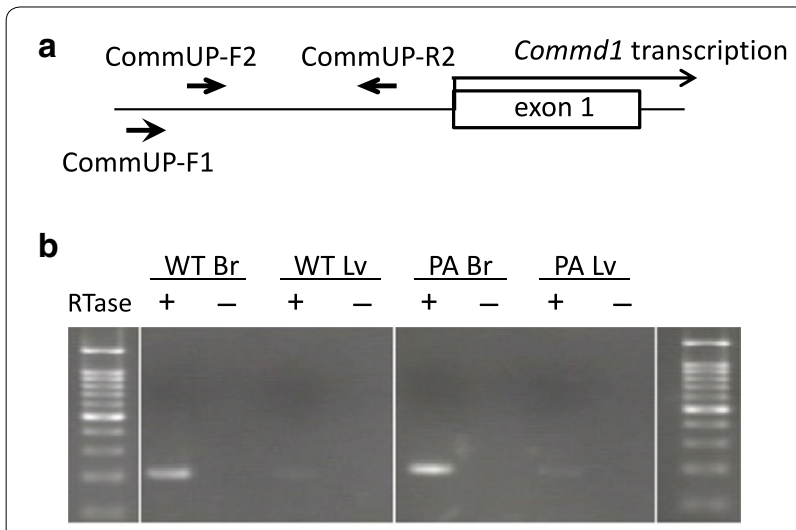

C

WT

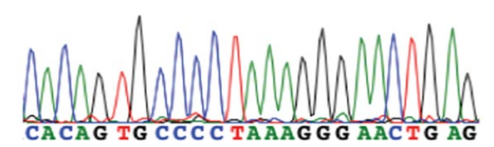

PA

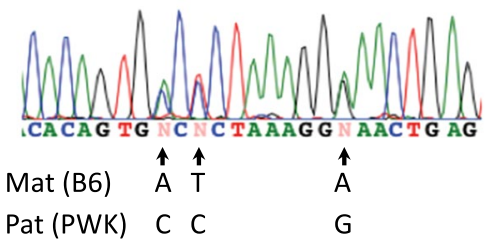

Fig. 5 The antisense transcript in the promoter region of Commd 1 in adult tissues. a Strand-specific RT-PCR was performed to detect an antisense transcript to Commd 1 transcription in the promoter region. The arrows represent the primers, CommUP-F1, for CDNA synthesis, and CommUP-F2 and CommUP-R2, for subsequent RT-PCR The amplicon is $206 \mathrm{bp}$ in size. CommUP-R2 is located approximately 100-bp upstream from the putative transcription start site of Commd1. b Brain (Br) and liver (Lv) RNA were analyzed. Tissues were prepared from adult F1 mice generated from crossing PA-B6 females with WT PWK males. CDNA synthesis was performed with (RTase +) or without (RTase -) reverse transcriptase. MW: molecular weight marker. c Allelic expression of the antisense transcripts was analyzed via direct sequencing of the amplified brain cDNA from the WT and PA mice shown in $\mathbf{b}$. Three SNPs used to discriminate the parental alleles are shown under the electropherograms

maternal expression in the $\triangle \mathrm{PA}$ mice. Moreover, the relative quantities of the overall expression were consistent with the quantitative allelic expression analysis shown in Fig. 4d, in which the total expression levels were 5 (maternal $=4$, paternal $=1$ ) and 3 (maternal $=2$, paternal=1) in the $\mathrm{WT}$ mice and the $\triangle \mathrm{PA}$ mice, respectively, given no change in the level of expression from the paternal allele. Collectively, these results indicate that expression of $\mathrm{Zrsr} 1$ reduces the expression of Commd1 from the paternal allele and results in the predominant maternal expression of Commd1.

In the Commd1 promoter region, we analyzed antisense transcription of Commd1 in F1 mice generated from the cross between Commd1 $1^{+/ P A}$ B6 females and
WT PWK males. Antisense transcripts were expressed at higher levels in the brain than in the liver of the WT mice, as reported previously [24], and the same transcript was observed in the PA mice (Fig. 5b). In addition, the antisense transcript was expressed only from the paternal allele in the WT mice, which is congruent with previous studies [24]. However, it was expressed from both alleles in the PA mice (Fig. 5c), which was consistent with the pattern of allelic expression of Zrsr1 in these mice. This consistency of allelic expression between the opposite transcripts and Zrsr1 strongly supports the idea that to some degree the transcription of Zrsrl extends to the Commd1 promoter region beyond the poly(A) signal of the Zrsr1 gene. Such antisense transcription might interfere with the initiation of Commd1 transcription and thereby contribute to its predominant maternal expression, especially in the brain.

\section{Discussion}

The results of this study indicate that transcription through the Zrsr1-DMR is essential for the establishment of methylation at the DMR in the growing oocyte, as reported for four other imprinted loci [13, 16-18]. Our results also indicate that transcription-dependent methylation in the Zrsr1-DMR does not occur after fertilization, which suggests that the mechanism of de novo methylation of the Zrsr1-DMR is oocyte-specific. We also found that paternally imprinted expression of Zrsr1 resulted in predominant maternal expression of Commd1, which is likely caused by transcriptional interference between Zrsr1 and Commd1 on the paternal allele.

The mouse embryo undergoes genome-wide de novo DNA methylation during the post-implantation period, at a level comparable to the growing oocyte [19, 25, 26]. In fact, the early embryo expresses factors essential for de novo DNA methylation, such as Dnmt3a, Dnmt3b, and Dnmt3l [28-30]. In this respect, the de novo DNA methylation activity in the pluripotent cells of the early embryo is comparable to that in the growing oocyte. However, our results indicated that methylation at Zrsr1DMR does not occur on either parental allele in the early embryo, despite Commd1 expression from both alleles (Fig. $4 a-c)$. This suggests some differences in the mechanisms of de novo methylation between the early embryo and the growing oocyte, at least in Zrsr1-DMR. Two possible differences are considered: first, there may be factor(s) inhibiting de novo methylation at Zrsr1-DMR in the early embryo, e.g., histone modification(s) at the DMR, or a trans-acting factor(s) expressed in pluripotent cells; second, the early embryo may lack factor(s) required for de novo methylation at the DMR, which could include epigenetic modification(s) at the DMR that are erased after 
fertilization, or trans-acting oocyte-specific factor(s). In contrast to the methylation at Zrsr1-DMR, transcriptiondependent methylation occurs during embryonic development at sDMRs located upstream of $Z d b f 2$, which is an imprinted gene on the paternal allele, during the period around implantation [31]. This suggests variation in DNA methylation mechanisms among DMRs in the imprinted loci, and it will be interesting to examine whether transcription-dependent methylation is also oocyte-specific at the four previously reported loci.

Several mechanisms are known or proposed for imprinted gene silencing, such as by allele-specific inactivation of an enhancer-insulator residing in a gDMR and by recruitment of repressive chromatin modifiers through a long non-coding RNA with imprinted expression $[2,4]$. Methylation of a gene promoter usually represses expression of the respective gene $[32,33]$. Thus, the paternal allele-specific expression of Zrsr1 seems to be caused by maternal methylation at Zrsr1-DMR. This was confirmed by analyses of the expression and methylation of Zrsr 1 in the PA mice, in which the absence of methylation at the maternal Zrsr1-DMR resulted in equal levels of gene expression from both alleles (Fig. 3c, d). A decrease in maternal expression of Commd1 also occurred concomitantly with the activation of maternal $\mathrm{Zrsr}$, which strongly suggests that the predominant maternal expression of Commd1 results from Zrsr1-mediated reduction in the expression of Commd1 from the paternal allele. Studies on genetic organization in genomes have identified overlapping and antisense-oriented genes, and the mutual cis-acting effect on their expression, which is termed transcriptional interference [34]. The active transcription of Zrsr1 within Commd1 may cause collisions between the opposing Zrsr1 and Commd1 elongation complexes, which would result in the reduction in their expression. We observed an antisense-oriented transcript in the Commd1 promoter region, which was hypothesized to be an overshoot of Zrsr1 transcription (Fig. 5) and suggests that Zrsr1 transcription potentially interferes with the initiation of Commd1 transcription. However, complete biallelic $Z r s r 1$ expression did not give rise to equal levels of Commd 1 expression from both parental alleles (Fig. 4d). Therefore, unidentified differences between the parental Commd1 alleles may exist, despite the fact that thus far we have found no differences in DNA methylation or some histone modifications in our examination of the Commd1 promoter [27].

Our results implicate the existence of unidentified growing oocyte-specific factors for the establishment of methylation at maternal gDMRs. We have yet to determine whether these factors are proteins, RNAs, or chromatin structures. Future extensive proteomic, transcriptomic, and epigenetic analyses of the growing oocyte may elucidate the underlying mechanism for the primary establishment of methylation at the maternal gDMRs.

\section{Conclusions}

We have found that Commd1 was expressed and thus the Zrsr1-DMR was transcribed in growing oocyte during the establishment of methylation at the DMR. Our data demonstrate that the transcription through the Zrsr1DMR is essential for the establishment of methylation at the DMR in the growing oocyte. Since methylation at the Zrsr1-DMR does not occur during embryonic development and postnatal growth, the mechanism of the DMR methylation is suggested to be oocyte-specific.

The imprinted paternal expression of Zrsr1 is caused by the maternal methylation at the Zrsr1-DMR which represses the maternal allele expression. The paternal expression of Zrsr1 results in predominant maternal expression of Commd1, likely caused by transcriptional interference between $\mathrm{Zrsr} 1$ and Commd1 on the paternal allele.

\section{Methods}

\section{Generation of Commd1-PA mice}

A truncation cassette was constructed by cloning three copies of the SV40 poly(A) signal from the expression vector pGFP-N1 (Clontech) into pT7Blue (Novagen). The truncation cassette was inserted at the genomic site $23 \mathrm{bp}$ downstream of exon 1 using the gene-targeting method (Fig. 1a). The targeting construct was generated by homologous recombination with a truncation cassette clone and a mouse Commd1 BAC clone (BAC RP24216A32, BACPAC Resources) in E. coli as previously described [35]. The targeting construct contained the following mouse Commd1 genomic sequences: a $5.2-\mathrm{kb}$ $5^{\prime}$ sequence containing exon 1 and a 5.1-kb $3^{\prime}$-sequence containing a part of intron 1 . An embryonic stem cell (ES) clone with the truncation cassette inserted in the precise genomic position was identified by Southern blotting and PCR analyses of genomic structure and was used to generate chimeric Commd1-PA mice. The neo gene in the targeting vector was flanked by FRTs and removed from the Commd1-truncated mice via Flippase expression. Commd1-PA mice in a C57BL/6 J (denoted B6) genetic background were obtained by backcrossing with wild type (WT) C57BL/6 J mice for five generations.

\section{Preparation of primordial germ cells, metaphase II oocytes, and blastocysts}

Mouse female primordial germ cells (PGC) were prepared from Day 5, Day 10, and Day 15 female C57BL/6 neonates as previously described [36]. The metaphase II (MII) oocytes were obtained from superovulated 
six- to eight-week-old female mice using the procedure described by Nakagata et al. [37]. Blastocysts were prepared by in vitro fertilization (IVF) using MII oocytes from Commd1 $1^{\mathrm{PA} /+} \mathrm{B} 6$ mice and sperm from 12- to 14-week-old CAG-Cre transgenic BALB/c mice. After cumulus-oocyte complexes had been coincubated with sperm for $3 \mathrm{~h}$, fertilized oocytes were cultured to the blastocyst stage at $37{ }^{\circ} \mathrm{C}$ and $5 \% \mathrm{CO}_{2}$ in humidified air for 96-120 h, as previously described [24]. CAG-Cre BALB/c mice were obtained from the RIKEN BioResource Center (RBRC No. RBRC06155). The CAG promoter drives ubiquitous expression of Cre recombinase in mice.

\section{DNA preparation and methylation analysis}

Approximately 200 growing oocytes and MII oocytes were lysed in lysis buffer $(0.5 \% \mathrm{SDS}, 250 \mathrm{ng} / \mu \mathrm{l}$ proteinase $\mathrm{K}, 100 \mathrm{ng} / \mu \mathrm{l}$ yeast tRNA) at $37^{\circ} \mathrm{C}$ for $60 \mathrm{~min}$, and DNA in the lysate was bisulfite-converted by treating the lysate with the EpiTect Bisulfite Kit (QIAGEN, \#59104). Recovered DNA was used for amplification of Zrsr1-DMR via nested PCR. Amplified DNA was cloned in pT7Blue T-vector (Novagen, \#69820), and the resulting sequences were analyzed with BigDye Terminator v3.1 (Applied Biosystems, \#4337455) on an ABI3130 sequencer (Applied Biosystems). Blastocyst DNA was prepared from the precipitate of ISOGEN II lysate during RNA preparation with ISOGENOME (NIPPON GENE, \#318-08111). DNA from adult tissues was prepared using QIAamp DNA Mini Kit (QIAGEN, \#51306). Blastocyst DNA was genotyped by genomic PCR, using primers on both sides of the truncation cassette (Fig EV2). DNA from blastocysts and tissues was also bisulfite-converted using the EZ DNA Methylation Kit (Zymo Research Corp., \#D5001). PCR, cloning, and sequencing were performed as described for oocyte DNA.

\section{RNA preparation}

RNA was prepared from approximately 100 growing and MII oocytes using the RNeasy Micro Kit (QIAGEN, \#74004). For RNA preparation from blastocysts and adult tissues, samples were lysed with ISOGEN II (NIPPON GENE, \#311-07361), and the cleared lysates were recovered by centrifugation according to the manufacturer's instructions. Three blastocysts were pooled for RNA preparation, and the lysate was loaded onto a spin column from the RNeasy Mini Kit (QIAGEN, \#74104). DNase I treatment, column wash, and RNA elution were performed according to the manufacturer's instructions.

\section{Expression analysis}

To analyze Commd1 expression in oocyte and adult tissues, cDNA was synthesized with random primers (Takara, \#3802) and reverse transcriptase (TOYOBO, TRT-101), and the Commd1 cDNA was amplified by PCR with the primers Comm-F1 (exon 1) and CommR1 (exon 2). To analyze allelic expression, restriction fragment length polymorphism (RFLP) analysis was performed via NlaIII digestion of the amplified cDNA. Allelic expression of Zrsr1 was analyzed by BigDye terminator sequencing of the cDNA amplified with the primers Zrsr-F1 and Zrsr-R1. No rs numbers were assigned to the single nucleotide polymorphisms (SNPs) that were used to discriminate between the C57BL/6 J and PWK alleles. Quantitative allelic expression analysis of Commd1 in blastocyst and adult tissues was performed in triplicate by pyrosequencing with PyroMark Q24 (QIAGEN) in the AQ assay mode. The rs26846230 SNP was used to discriminate between the C57BL/6 J and BALBc alleles. Total expression levels were quantitated in triplicate using the StepOnePlus real-time PCR system with TaqMan Gene Expression Assay (Applied Biosystems) Mm00495837_s1 and Mm01239669_m1 for Zrsr1 and Commd1, respectively. Actb ( $\beta$-actin) mRNA was quantitated as an internal control using TaqMan Assay Mm00607939_s1.

\section{Primers}

All primers used in this study are listed in Additional file 4: Table S1.

\section{Additional files}

Additional file 1: Figure S1. RT-PCR analysis of Zrsr1 expression in growing oocytes. RT-PCR was done with primers Zrsr-F1 and Zrsr-R1 using cDNAs in Fig. 1b. Growing oocytes were prepared from B6 female neonates at Day 5 (D5), Day 10 (D10), and Day 15 (D15) postpartum, and fully grown MII oocytes (MII) from B6 adult females. PC: positive control for RT-PCR using adult brain CDNA. MW: molecular weight marker. Two different CDNA batches were used for D5 RNA.

Additional file 2: Figure S2. Genotyping PCR of blastocysts carrying Commd ${ }^{P A}$ and CAG-Cre transgene. A Schematic representation of PCR for three Commd 1 alleles, Commd1 ${ }^{P A}(\mathrm{PA}), \mathrm{Commd} 1^{\mathrm{DPA}}$ (DPA) and Commd $1^{+}$ (WT). B Electrophoresis of PCR products of nine blastocysts positive for Commd $1^{P A}$ and CAG-Cre transgene among 24 blastocysts obtained from an IVF performed with oocytes from PA female mice and sperm from CAG-Cre male mice. Two blastocysts (\#5, \#9) contained small amount of undeleted truncation cassette. MW: molecular weight marker.

Additional file 3: Figure S3. Quantitative analysis of the allelic expression of Commd 1 in adult mice. The allelic expression was quantitatively analyzed by pyrosequencing. The levels of expression from the parental alleles are shown relative to the level of the paternal allele (1.0). Each sample was analyzed in triplicate. A Brains and livers from two WT adult F1 mice between B6 females and PWK males. B Brains and livers from three WT adult F1 mice between B6 females and BALBC males.

Additional file 4: Table S1. List of the primers used in this study. 


\section{Abbreviations}

CDNA: complementary DNA; DMR: differentially methylated region; mRNA: messenger RNA; PCR: polymerase chain reaction; RT-PCR: reverse transcription PCR

\section{Authors' contributions}

$\mathrm{KJ}$ designed and carried out most of the experiments. KN and HK contributed the generation of PA mice. FM, SK, and TK contributed materials. HY and KH contributed analysis tools. HS obtained funding, designed experiments, and oversaw the research. $\mathrm{KJ}$ and $\mathrm{HS}$ wrote the manuscript. All authors read and approved the final manuscript.

\section{Author details}

${ }^{1}$ Division of Molecular Genetics and Epigenetics, Department of Biomolecular Sciences, Faculty of Medicine, Saga University, Saga 849-8501, Japan. ${ }^{2}$ Division of Biological Resources and Development, Analytical Research Center for Experimental Sciences, Saga University, Saga 849-8501, Japan. ${ }^{3}$ Laboratory of Animal Developmental Biology, Department of Bioscience, Faculty of Applied Biosciences, Tokyo University of Agriculture, Tokyo 156-8502, Japan. ${ }^{4}$ Laboratory for Developmental Genetics, RIKEN Center for Integrative Medical Science, Yokohama, Kanagawa 230-0045, Japan.

\section{Acknowledgements}

We thank Dr. Tatsuya Kishino from the Gene Research Center, Center for Frontier Life Sciences, Nagasaki University, for providing the PWK mice used in this study.

\section{Competing interests}

The authors declare that they have no competing interests.

\section{Availability of data and materials}

All data generated or analyzed during this study are included in this published article. All materials generated during the current study are available from the corresponding author on reasonable request.

\section{Consent for publication}

Not applicable.

\section{Ethics approval and consent to participate}

This study was approved by the Ethical Committee for Animal Experiment of Saga University (approval \# 28-032-0).

\section{Funding}

This study was supported in part by Grants-in-Aid for Scientific Research (C) from the Japan Society for the Promotion of Science [16K09970 to K.H., $17 K 08687$ to H.S.]; grants for Practical Research Project for Rare/Intractable Diseases from the Japan Agency for Medical Research and Development (AMED) [17ek0109280h0001, 17ek0109234h0001, 17ek0109205h0001]; a grant for Child Health and Development from the National Center for Child Health and Development [26-13]; a grant for Research on Intractable Diseases from the Ministry of Health, Labor, and Welfare [H29-nanchitou(nan)-ippan-025] and the joint research program of the Institute for Molecular and Cellular Regulation, Gunma University [16029 to K.H.].

\section{Publisher's Note}

Springer Nature remains neutral with regard to jurisdictional claims in published maps and institutional affiliations.

\section{Received: 9 March 2018 Accepted: 31 May 2018}

Published online: 06 June 2018

\section{References}

1. Arnaud P. Genomic imprinting in germ cells: imprints are under control. Reproduction. 2010;140:411-23.

2. Ferguson-Smith AC. Genomic imprinting: the emergence of an epigenetic paradigm. Nat Rev Genet. 2011;12:565-75.

3. Kobayashi H, Sakurai T, Sato S, Nakabayashi K, Hata K, Kono T. Imprinted DNA methylation reprogramming during early mouse embryogenesis at the Gpr1-Zdbf2 locus is linked to long cis-intergenic transcription. FEBS Lett. 2012;586:827-33.

4. Barlow DP, Bartolomei MS. Genomic imprinting in mammals. Cold Spring Harb Perspect Biol. 2014;6:a018382.

5. Wang L, Zhang J, Duan J, Gao X, Zhu W, Lu X, Yang L, Li G, Ci W, Li W, et al. Programming and inheritance of parental DNA methylomes in mammals. Cell. 2014:157:979-91.

6. Bartolomei MS, Ferguson-Smith AC. Mammalian genomic imprinting. Cold Spring Harb Perspect Biol. 2011:3:a002592.

7. Hata K, Okano M, Lei H, Li E. Dnmt3L cooperates with the Dnmt3 family of de novo DNA methyltransferases to establish maternal imprints in mice. Development. 2002;129:1983-93.

8. Kaneda M, Okano M, Hata K, Sado T, Tsujimoto N, Li E, Sasaki H. Essential role for de novo DNA methyltransferase Dnmt3a in paternal and materna imprinting. Nature. 2004;429:900-3.

9. Bourc'his D, Xu GL, Lin CS, Bollman B, Bestor TH. Dnmt3L and the establishment of maternal genomic imprints. Science. 2001;294:2536-9.

10. Ciccone DN, Su H, Hevi S, Gay F, Lei H, Bajko J, Xu G, Li E, Chen T. KDM1B is a histone $\mathrm{H} 3 \mathrm{~K} 4$ demethylase required to establish maternal genomic imprints. Nature. 2009:461:415-8.

11. Nashun B, Hill PW, Smallwood SA, Dharmalingam G, Amouroux R, Clark SJ, Sharma V, Ndjetehe E, Pelczar P, Festenstein RJ, et al. Continuous histone replacement by Hira is essential for normal transcriptional regulation and de novo DNA methylation during mouse oogenesis. Mol Cell. 2015;60:611-25.

12. Kelsey G, Feil R. New insights into establishment and maintenance of DNA methylation imprints in mammals. Philos Trans R Soc Lond B Biol Sci. 2013;368:20110336.

13. Veselovska L, Smallwood SA, Saadeh H, Stewart KR, Krueger F, MaupetitMéhouas S, Arnaud P, Tomizawa S, Andrews S, Kelsey G. Deep sequencing and de novo assembly of the mouse oocyte transcriptome define the contribution of transcription to the DNA methylation landscape. Genome Biol. 2015:16:209.

14. Ball MP, Li JB, Gao Y, Lee JH, LeProust EM, Park IH, Xie B, Daley GQ, Church GM. Targeted and genome-scale strategies reveal gene-body methylation signatures in human cells. Nat Biotechnol. 2009;27:361-8.

15. Maunakea AK, Nagarajan RP, Bilenky M, Ballinger TJ, D'Souza C, Fouse SD, Johnson BE, Hong C, Nielsen C, Zhao Y, et al. Conserved role of intragenic DNA methylation in regulating alternative promoters. Nature. 2010;466:253-7.

16. Chotalia M, Smallwood SA, Ruf N, Dawson C, Lucifero D, Frontera M, James K, Dean W, Kelsey G. Transcription is required for establishment of germline methylation marks at imprinted genes. Genes Dev. 2009;23:105-17.

17. Smith EY, Futtner CR, Chamberlain SJ, Johnstone KA, Resnick JL. Transcription is required to establish maternal imprinting at the Prader-Willi syndrome and Angelman syndrome locus. PLoS Genet. 2011;7:e1002422.

18. Singh VB, Sribenja S, Wilson KE, Attwood KM, Hillman JC, Pathak S, Higgins MJ. Blocked transcription through KvDMR1 results in absence of methylation and gene silencing resembling Beckwith-Wiedemann syndrome. Development. 2017:144:1820-30.

19. Smallwood SA, Tomizawa S, Krueger F, Ruf N, Carli N, Segonds-Pichon A, Sato S, Hata K, Andrews SR, Kelsey G. Dynamic CpG island methylation landscape in oocytes and preimplantation embryos. Nat Genet. 2011:43:811-4

20. Monk D. Germline-derived DNA methylation and early embryo epigenetic reprogramming: the selected survival of imprints. Int J Biochem Cell Biol. 2015;67:128-38.

21. Nabetani A, Hatada I, Morisaki H, Oshimura M, Mukai T. Mouse U2af1-rs1 is a neomorphic imprinted gene. Mol Cell Biol. 1997;17:789-98.

22. Lucifero D, Mann MR, Bartolomei MS, Trasler JM. Gene-specific timing and epigenetic memory in oocyte imprinting. Hum Mol Genet. 2004;13:839-49.

23. van de Sluis B, Muller P, Duran K, Chen A, Groot AJ, Klomp LW, Liu PP, Wijmenga $C$. Increased activity of hypoxia-inducible factor 1 is associated with early embryonic lethality in Commd 1 null mice. Mol Cell Biol. 2007;27:4142-56.

24. Wang Y, Joh K, Masuko S, Yatsuki H, Soejima H, Nabetani A, Beechey CV, Okinami S, Mukai T. The mouse Murr1 gene is imprinted in the adult brain, presumably due to transcriptional interference by the antisenseoriented U2af1-rs1 gene. Mol Cell Biol. 2004;24:270-9. 
25. Howlett SK, Reik W. Methylation levels of maternal and paternal genomes during preimplantation development. Development. 1991;113:119-27.

26. Kafri T, Ariel M, Brandeis M, Shemer R, Urven L, McCarrey J, Cedar H, Razin A. Developmental pattern of gene-specific DNA methylation in the mouse embryo and germ line. Genes Dev. 1992;6:705-14.

27. Joh K, Yatsuki H, Higashimoto K, Mukai T, Soejima H. Antisense transcription occurs at the promoter of a mouse imprinted gene, commd1, on the repressed paternal allele. J Biochem. 2009;146:771-4.

28. Okano M, Bell DW, Haber DA, Li E. DNA methyltransferases Dnmt3a and Dnmt3b are essential for de novo methylation and mammalian development. Cell. 1999;99:247-57.

29. Watanabe D, Suetake I, Tada T, Tajima S. Stage- and cell-specific expression of Dnmt3a and Dnmt3b during embryogenesis. Mech Dev. 2002:118:187-90

30. Uysal F, Akkoyunlu G, Ozturk S. Dynamic expression of DNA methyltransferases (DNMTs) in oocytes and early embryos. Biochimie. 2015;116:103-13.

31. Greenberg MV, Glaser J, Borsos M, Marjou FE, Walter M, Teissandier A, Bourc'his D. Transient transcription in the early embryo sets an epigenetic state that programs postnatal growth. Nat Genet. 2017:49:110-8.
32. Bird A. DNA methylation patterns and epigenetic memory. Genes Dev. 2002;16:6-21.

33. Esteller M. Cancer epigenomics: DNA methylomes and histone-modification maps. Nat Rev Genet. 2007;8:286-98.

34. Bordoy AE, Chatterjee A. Cis-antisense transcription gives rise to tunable genetic switch behavior: a mathematical modeling approach. PLOS ONE. 2015;10:e0133873.

35. Zhang Y, Muyrers JP, Testa G, Stewart AF. DNA cloning by homologous recombination in Escherichia coli. Nat Biotechnol. 2000;18:1314-7.

36. Kobayashi H, Sakurai T, Miura F, Imai M, Mochiduki K, Yanagisawa E, Sakashita A, Wakai T, Suzuki Y, Ito T, et al. High-resolution DNA methylome analysis of primordial germ cells identifies gender-specific reprogramming in mice. Genome Res. 2013;23:616-27.

37. Nakagata N, Takeo T, Fukumoto K, Kondo T, Haruguchi Y, Takeshita Y, Nakamuta Y, Matsunaga H, Tsuchiyama S, Ishizuka Y, Araki K. Applications of cryopreserved unfertilized mouse oocytes for in vitro fertilization. Cryobiology. 2013;67:188-92.
Ready to submit your research? Choose BMC and benefit from:

- fast, convenient online submission

- thorough peer review by experienced researchers in your field

- rapid publication on acceptance

- support for research data, including large and complex data types

- gold Open Access which fosters wider collaboration and increased citations

- maximum visibility for your research: over 100M website views per year

At BMC, research is always in progress.

Learn more biomedcentral.com/submissions 